REVIEW ARTICLE

\title{
Enhanced Liver Fibrosis (ELF) Immunoassay Test for Identification of Significant Liver Diseases: Recent Developments
}

\author{
RS Giasuddin ${ }^{1}$, M Khan $^{2}, *$ AS M Giasuddin ${ }^{3}$, MMR Siddiqui $^{4}$ \\ ${ }^{1}$ Dr. Rubayat Sheik Giasuddin, Assistant Professor of Medicine, Department of Medicine, Anwar Khan Modern \\ Medical College and Hospital, \\ ${ }^{2}$ Prof. Dr. Mobin Khan, Former Professor of Hepatology, Department of Hepatology \\ Bangabandhu Sheikh Mujib Medical University (BSMMU). \\ 3*Prof. Dr. ASM Giasuddin, Professor of Biochemistry \& Immunology, Dept of Medical Laboratory Science, \\ State College of Health Sciences \& Adjunct Professor, State University of Bangladesh, \\ ${ }^{4}$ Dr. Md. Mahmudur Rahman Siddiqui, Assistant Professor of Medicine, Department of Medicine, Anwar Khan \\ Modern Medical College and Hospital \\ *Corresponding Author
}

\begin{abstract}
Liver Fibrosis (LF) is a common outcome in chronic liver disease (CLD). Over a period of time, the process results in cirrhosis leading to disruption in functional capacity of liver. Further serious complications may occur including portal hypertension, liver failure and cancer. The magnitude of CLD has increased being recognized as a major cause for morbidity and mortality globally and it is increasing progressively in Bangladesh also. Question arises "Is the transabdominal needle biopsy of liver, the traditional reference standard method, sufficient and efficient enough for detecting and assessing liver fibrosis particularly in the early stages of significant liver disease?" More simpler, convenient and less expensive blood tests such as 'Enhanced Liver Fibrosis (ELF) immunoassay test', Fibrotest and Aspartate transaminase-platelet ratio index (APRI), have been developed and reported for identification of significant CLD and use as prognostic tool in clinical practice. Among them, the ELF test had been found to be statistically the best for diagnosing and predicting clinical outcomes in patients with CLD and may be a useful prognostic tool in clinical practice. This ELF immunoassay test has been briefly reviewed in this article. Short title: ELF immunoassay test for CLD
\end{abstract}

Key Words: Immunoassay test, Chronic liver disease, Liver fibrosis

\section{Introduction}

Chronic liver disease (CLD), resulting from viral hepatitis, fatty liver or alcoholic liver disease, is increasingly recognized as a major cause of morbidity and mortality globally $1,2,3,4,5$. The magnitude of liver diseases ranging from acute viral hepatitis, which is more commonly familiar as jaundice, to liver cancer is increasing progressively in Bangladesh ${ }^{6,7}$. Although HAV and HEV remained a constant problem throughout the world, HBV and HCV are the commonest culprits causing CLD leading to cirrhosis and finally liver cancer. Furthermore, consumption of adulterated foods, fruits, edible oils, etc may be causative factors for long term inflammation leading to fibrosis and finally CLD such as cirrhosis and cancer ${ }^{8,9}$.

There is a deep concern for future generation of Bangladesh for their increased habit forwards so called fast food tradition which contains excess fat and other noxious agents leading to fatty changes in the liver and progression to CLD in the long run. In comparison to the developing countries, liver diseases are more common in Bangladesh due to the aforesaid factors 6,7 . Therefore, highly efficient, convenient and relatively cheaper tests for diagnosis and prognosis which are more informative and acceptable to the patients globally should be made available.

\section{Fibrosis}

Fibrosis, a common outcome in CLD, is the accumulation of fibrous connective tissue in the liver. The formation of this 'scar' tissue through the deposition of new collagen is a normal bodily response to injury, but in fibrosis this healing process goes faulty without control. Over a period of time, this process results in cirrhosis of liver in which the functional capacity of the liver is disrupted (Figure-1) ( $^{8,9,10}$. 


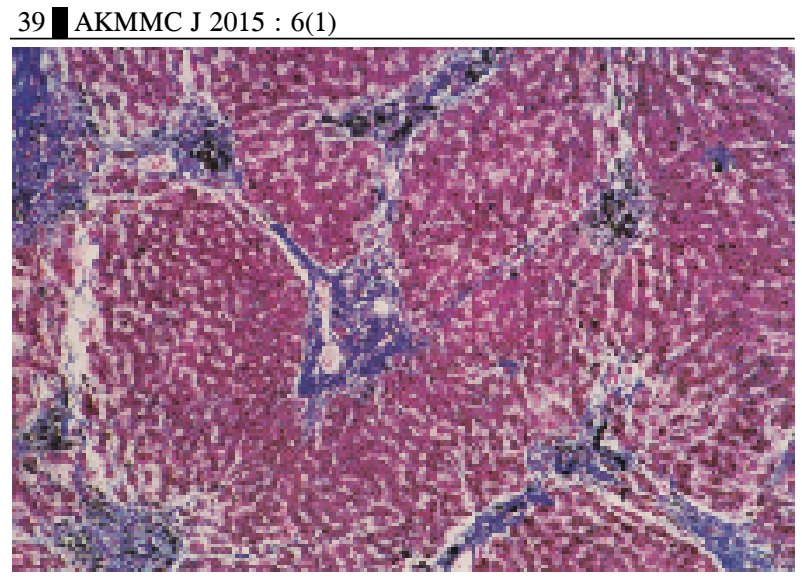

Figure-1: Light micrograph of a section of human liver showing the typical appearance of cirrhosis. Bands of fibrous tissue (blue) can be seen to disrupt the arrangement of hepatocytes Adopted from ref no. 10).

Further serious complications may occur including portal hypertension, liver failure and cancer. Cirrhosis is considered to be a premelignant condition and greatly increases the risk of liver cancer. Cirrhosis and liver cancer are among the top 10 causes of death worldwide ${ }^{11,12}$.

$\mathrm{HCV}$ and HBV infections have contributed greatly to the burden to alcoholic liver disease (ALD), over the past few decades in many developed as well as developing cerentries. In the future this may result in an epidemic of liver fibrosis due to the vast size of the at-risk population. Question therefore arises "Is the transabdominal needle biopsy of liver, the traditional reference standard method, sufficient and efficient enough for detecting and assessing liver fibrosis particularly in the early stages of significant liver disease $\mathrm{s}^{9,13,14}$ ?". Certainly more simpler, convenient and less expensive procedures would be much appreciated and useful. Only recently simpler blood tests such as Enhanced liver fibrosis (ELF) immunoassay test, Fibrotest and Aspartate transaminase-platelet ratio index (APRI) have been developed and reported for identification of significant CLD and use as prognostic tool in clinical practice ${ }^{8,9}$. This ELF- immunoassay test has been briefly reviewed in this article.

\section{Liver Biopsy \& its Limiations}

The traditional reference standard procedure, i.e. transabdominal needle biopsy of liver, is routinely performed to assess liver damage (fibrosis) and to try to monitor effectiveness of treatment. Small sample size and the patchy distribution of some liver pathology can result in a significant degree of
S Giasuddin, M Khan, AS M Giasuddin et al

sampling error. Also, the procedure is hazardous, with bleeding in approximately 01 out of 1000 cases and death in 01 out of 10,000 cases. Histological examination of the biopsy is time-consuming and subject to inter-observer variability. Thus, this method of assessment of fibrosis is a poor and inadequate reference standard. Furthermore, biopsy is unsuitable for repeat testing and cannot easily be used to monitor disease progression or response to treatement. ${ }^{13,14}$

\section{Non-Invasive Markers of Liver Fibrosis}

Non-invasive markers for liver fibrosis are the best possible alternatives, can offer significant advantages and benefits compared to biopsy. The available serum markers can be classified into indirect and direct markers.

\section{Indirect serum markers of liver fibrosis include the following:}

Markers released into the blood as a result of liver inflammation, eg. Alanine aminotransferase (ALT), Aspartate animotransferase (AST) and Gammaglutamyl transferase $(\gamma$-GT); makers synthesised, regulated or excreted by the liver, eg. clotting factors, cholesterol and bilirubin; processes that become disturbed by impaired liver function, eg. insulin resistance.

\section{Direct serum markers of liver fibrosis include the following:}

Direct serum markers of fibrosis are those molecules involved in fibrosis regulation together with fragments of liver matrix components produced during the fibrotic process. Liver fibrosis is now recognized as a dynamic process that can be progressive or regressive over a period of months. Fibrosis tissue remodeling occurs through digestion by matrix metalloproteinases (MMPs). This digestion process is controlled through the inhibition of MMPs by tissue inhibitors of MMPs (TIMPs). Among these TIMPs, TIMP-1 is of major importance. Therefore, direct serum markets of fibrosis include the following:

Hyaluronic (HA), Collagens IV \& VI, Procollagen 3 amino terminal peptide (P3NP), Matrix mtalloproteinases (MMPs) and Tissue inhibitor of MMP1 (TIMP-1).

Recent studies have indicated that ELF markers are as good as and often better than liver histopathology at predicating clinical outcome $e^{10,13,14,15,16}$. 
Enhanced Liver Fibrosis (ELF) Immunoassay Test for Identification Enhanced Liver Fibrosis (Elf) Immunoassay Test

The combination of three direct serum markers, i.e. HA, P3NP and TIMP-1 in an algorithm, can be used to determine accurately the extent and severity of fibrosis. This combined three blood tests together is the ELF immunoassay test, which is now fully $\mathrm{v}$ a $\mathrm{l}$ i d a $\mathrm{t}$ e d , C E - m a r k e d standardised and made commercially available as a testing service for patient management ${ }^{15,17,18,19}$.

\section{Severity, Progression \& Regression Assessment}

Several studies have confirmed that the ELF test results accurately reflects the severity of fibrosis as staged on liver biopsy.

Friedrich-Rust et al analyzed ELF-test (consisting of an algorithm of three fibrosis markers i.e. HA, P3NP, TIMP1) retrospectively in patients with CLD who received a liver biopsy, transient elastography and the FibroTest using histology as the reference method. They reported that the area under the reviewer operator characteristic curve (AUROC) was highest for ELF test, i.e. 0.78 (95\% CI: 0.67-0.89) and that FibroTest and ELF can be performed with comparable diagnostic accuracy for the non-invasive staging of liver fibrosis. They also concluded that serum tests are informative in a higher proportion of patients than transient elastography ${ }^{20}$. Parkes et al followed up 457 patients recruited for ELF study (median 7 years) for liver-related morbidity and mortality and reported that patients having the highest ELF scores (12.52-16.67) being significantly(p 0.05) more likely to have clinical outcomes (deaths) than those in lower score groups (8.3412.51) compared to patients with ELF score $<8.34^{21}$.

Lichtinghagen et al identified three cut-off values: 7.7 for a high sensitivity exclusion of fibrosis, 9.8 for a high specificity identification of fibrosis (sensitivity $69 \%$, specificity $98 \%$ for moderate fibrosis), and 11.3 to discriminate cirrhosis (sensitivity $83 \%$, specificity 97\%). They found the ELF score validity was superior to the results of the single tests. They concluded that the ELF score can predict moderate fibrosis and cirrhosis although influence factors such as gender and age need to be taken into consideration ${ }^{22}$.

Recently, Xie et al identified nine studies to perform a meta-analysis to assess the performance of ELF test for the assessment of liver fibrosis. They reported that the pooled sensitivity, specificity, positive LR, negative LR, and DOR values of ELF test, for assessment of significant liver fibrosis, were $83 \%$ ( $95 \% \mathrm{CI}=0.80-0.86$ ), $73 \%$ (95\% CI=0.69-0.77), $4.00(95 \% \mathrm{CI}=2.50-6.39)$, $0.24(95 \% \mathrm{CI}=0.17-0.34)$, and $16.10(95 \% \mathrm{CI}=8.27-$
31.34), respectively; and, for evaluation of severe liver fibrosis, were $78 \%$ (95\% CI $=0.74-0.81), 76 \%(95 \%$ $\mathrm{CI}=0.73-0.78), 4.39(95 \% \mathrm{CI}=2.76-6.97), 0.27(95 \%$ $\mathrm{CI}=0.16-0.46)$, and 16.01 (95\% CI: $7.15-35.82)$, respectively; and, for estimation of cirrhosis, were $80 \%$ (95\% CI $=0.75-0.85), 71 \%(95 \% \mathrm{CI}=0.68-0.74), 3.13$ $(95 \% \mathrm{CI}=2.01-4.87), 0.29(95 \% \mathrm{CI}=0.19-0.44)$, and 14.09 (95\% CI: 5.43-36.59), respectively. The ELF test showed good performance and considerable diagnostic value for the prediction of histological fibrosis stage ${ }^{23}$.

In these studies, AUROC is approximately 0.8 . This level of performance is considered to be a threshold for acceptance in clinical practice (Figure-2) ${ }^{10,21,23 .}$

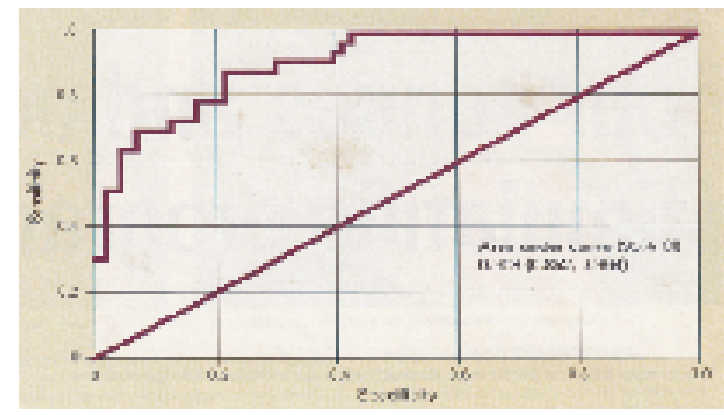

Figure-2: Area under the receiver operator characteristic cure (AUROC) for the ELF test, predicting stages 0-1 versus 2-6 in the NAFLD cohort (none or mild fibrosis from significant fibrosis (Adopted from ref no. 10).

Interim analysis of longitudinal studies indicates that ELF markers are as good as and often better than liver histopathology at predicting clinical outcome. Thus, ELF markers have great potential in the screening and management of patients with CLD. In secondary care, the ELF test will be of great value in the early evaluation of patients with a wide range of CLD. The ELF test will not replace the liver biopsy in the detailed assessment of liver inflammation and architectural damage; However, repeated biopsy is not acceptable in the vast majority of patients. On the contrary, the ELF test can be repeated at frequent internals which is a significant advantage over biopsy $14,15,20,21,22,23$.

\section{Conclusions}

The ELF immunoassay test is the first standardized laboratory method for assessment of liver fibrosis together with its progression and regression. It requires only a sample of blood. Sampling for ELF test can be performed in a physician's chamber or health centre, avoiding the need for a patient to travel to a hospital. The ELF test, which can be repeated at frequent intervals, will enable more accurate assessment of the severity of fibrosis together with its progression or regression in response to treatments. In 
addition, this ELF test is expected to be more acceptable to the patients in developing and poor countries as well. In conclusion, the ELF test showed good performance, considerable diagnostic value for the histological fibrosis stage, can predict clinical outcome in patients with CLD and may be a useful prognostic tool in clinical practice.

It is a matter of delight that this ELF immunoassay test is available commercially as a service for physicians managing patients with CLD. However, true potential of the ELF immunoassay test may not be realised until further longitudinal studies serially measuring the serum markers against clinical outcomes are published.

\section{Acknowledgements}

The technical assistance of Mr Kazim Kamran, IT Section, State College of Health Sciences, Dhanmondi, Dhaka-1209, is highly appreciated.

\section{Conflict of Interest}

The authors have no competing or conflict of interest.

\section{References}

1. Longo DL, Fauci AS, Kasper DL et al (Editors). Harrison's Principles and Practice of Internal Medicine, 18th Edition; New York: McGraw Hill; 2013.

2. Walker BR, Colledge NR, Ralston $\mathrm{SH}$ et al (Editors). Davidson's Principles and Practice of Medicine, $22^{\text {nd }}$ Edition; Edinburgh: Churchill Livingstone Elsevier; 2014.

3. Kumar P, Clark ML (Editors). Kumar and Clark's Clinical Medicine, 8th Edition; Edinburgh: Elsevier Health Sciences; 2012.

4. Bosch FX, Ribes J, Diaz M, et al. Primary liver cancer; worldwide incidence and trends. Gastroenterology.2004; 127(5 Suppl 1): S5-S16.

5. Donato F, Tagger A, Gelatti U, et al. Alcohol and hepatocellular carcinoma: The effect of lifetime intake and hepatitis virus infections in men and women. Am J Epidemiol 2002; 155(4): 323-31.

6. Giasuddin RS, A study on actiological diagnosis and presenting features in adult patients with space occupying lesions in the liver. FCPS dissertation submitted to the Bangladesh College of Physicians and Surgeons, Dhaka, Bangladesh; 2011.

7. Bahirwani R, Reddy KR, The evaluation of solitary liver masses. Aliment Pharmacol Ther 2008 Oct; 28(8):953-65.

8. Liver and Biliary Tract. In: Kumar V, Abbas AK, Fausto $\mathrm{N}$ et al (Editors). Robbins and Cotran Pathologic Basis of Disease. $8^{\text {th }}$ Edition; New York: Elsevier; 2010; 1591-1708.

9. Bialecki ES, Ezenekwe AM, Brunt, Sin EM, et al. Comparison of liver biopsy and noninvasive methods for diagnosis of hepatocellular carcinoma. Clin Gastroenterol Hepatol 2006; 4: 361-68.
10. Clinical Chemistry Update. Identification of significant liver disease: a simple blood test. The Biomedical Scientist (Gazette of the institute of Biomedical Science) 2010 July; 54(7): 490-91.

11. Szklaruk J, Silverman PM, Charmsangavej C. Imaging in the diagnosis staging, treatment and surveillance of hepatocellular carcinoma. Am J Roentgenol 2003; 180: 441-54.

12. Kinkel K, Lu Y, Both M. Detection of hepatic metastases from cancers of the gastrointestinal tract by using noninvasive imaging methods (US, CT, MR imaging, PET): a meta-analysis. Radiology 2002; 224(3): 748-56.

13. Parkes J, Guha IN, Roderick P, et al. Enhanced liver fibrosis (ELF) test accurately identifies liver fibrosis in patients with chronic hepatitis C. J Viral Hepatitis. (doi; 10.1111/J. 13652893.2009. 01263.x) (Accessed on 24 July 2010).

14. Barciy L. Enhanced liver fibrosis panel aids testing for nonalcoholic fatty liver disease. Hepatology 2008; 47(2). 455-60.

15. The Hepatitis C Trust. Enhanced Liver fibrosis (ELF) test available in Europe. Medical News Today; 28 July 2007 (Accessed on 24 July 2010).

16. Lin T, Wang X, Karsdal MA, et al. Molecular serum markers of liver fibrosis. Biomark Insights 2012; 7:105117. doi: 10.4137/BMI. S10009.

17. http://www.siemens.co.uk/diagonstitics (Accessed on 24 July 2010).

18. http://www.iqur.com (Accessed on May 2014).

19. Knudsen CS, Heickendorff L, Nexo E, Measurement of amino terminal propeptide of type III procollagen (PIII NP) employing the ADVIA Centaur platform: Validation, reference interval and comparison to UniQ RIA. Clin Chem Lab Med 2014 Feb; 52(2): 237-41. doi: 0.1515/cclm-20130502 .

20. Friedrich-Rust M, Rosenberg W, Parkes J, et al. Comparison of ELF, FibroTest and FibroScan for the non-invasive assessment of liver fibrosis. BMC Gastroenterol 2010 Sep 9; 10:103. doi: 10.1186/1471-230X-10-103.

21. Parkes J, Roderick P, Harris $\mathrm{S}$, et al. Enhanced liver fibrosis test can predict clinical outcomes in patients with chronic liver disease. Gut 2010 Sep;59(9):1245-51. doi: 10.1136/gut.2009.203166. Epub 2010 Jul 30.

22. Lichtinghagen R, Pietsch D, Bantel H, et al. The Enhanced Liver Fibrosis (ELF) score: normal values, influence factors and proposed cut-off values. J Hepatol 2013 Aug; 59(2):23642. doi: 10.1016/j.jhep.2013.03.016. Epub 2013 Mar 21.

23. Xie Q, Zhou X, Huang $P$, et al. The performance of enhanced liver fibrosis (ELF) test for the staging of liver fibrosis: a meta-analysis. PLoS One 2014 Apr 15; 9(4):e92772. doi:10.1371/journal.pone.0092772. eCollection 2014. 